Ann N Y Acad Sci. 2010 January ; 1183: 13-24. doi:10.1111/j.1749-6632.2009.05128.x.

\title{
Thymic stromal lymphopoietin
}

\author{
Rui He and Raif S. Geha \\ Division of Immunology, Children's Hospital, Harvard Medical School, Boston, MA, 02115
}

\begin{abstract}
Thymic stromal lymphopoietin (TSLP) is an epithelial cell derived cytokine expressed in skin, gut, lungs and thymus. TSLP signals via TSLPR, a heterodimer of the IL-7 receptor alpha chain (IL-7R $\alpha$ ) and the TSLP receptor chain (TSLPR), which is closely related to the common receptor$\gamma$ chain $(\gamma c)$, expressed on a wide range of cell types in the adptaive and innate immune system. TSLP exerts profound influence on the polarization of dendritic cells (DCs) to drive Thelper (Th) 2 cytokine production. It also directly promotes $\mathrm{T}$ cell proliferation in response to $\mathrm{T}$ cell receptor (TCR) activation, and Th2 cytokine production. TSLP also supports B cell expansion and differentiation. TSLP further amplifies Th2 cytokine production by mast cells and NKT cells. These properties confer on TSLP a critical role in driving Th2 mediated inflammation. This role is supported by the finding that TSLP expression is up-regulated in keratinocytes of atopic dermatitis (AD) skin lesions and in bronchial epithelial cells in asthma.
\end{abstract}

\section{Introduction}

Epithelial cells represent the first line of defense of the organism against microbes and foreign antigens that come in contact with body surfaces such as skin, gut and airways. Recent work strongly suggests that cytokines produced by epithelial cells play a critical role in shaping the immune response and in the pathogenesis of immune mediated diseases.

This review focuses on the epithelial cell derived TSLP which is expressed in skin, gut, lungs and thymus. TSLP has generated a great deal of interest because it appears to be a master regulator of Thelper (Th) 2 driven inflammation. We review the structure of TSLP and its receptor, their expression and the mechanism of TSLP signaling. We then review the role of TSLP in driving the polarization of DCs to induce Th2 cell differentiation during the induction phase of the immune response and its direct actions on $\mathrm{T}$ cells which promote $\mathrm{T}$ cell expansion and amplify Th2 cytokine secretion. We review the data on the potential role of TSLP in the development of T regulatory (Treg) cell and the action of TSLP on innate immune cells. Finally, we review the evidence that TSLP plays an important role in allergic diseases that include asthma and atopic dermatitis (AD) and its potential protective role in inflammatory bowel disease (IBD).

\section{TSLP and its receptor \\ Structure of TSLP and TSLPR}

TSLP is an epithelially derived cytokine initially identified in 1994 as a bioactive factor secreted in the supernatants of a murine thymic stromal cell line ${ }^{1}$. TSLP is a four-helix bundle cytokine, and is closely related to IL-7, with which it shares an overlapping, but distinct, biological profile. The mouse TSLP gene is located on chromosome 18, and the mature mouse

Correspondence to Raif S. Geha, Division of Immunology, Children's Hospital, One Blackfan Circle, Boston, Massachusetts 02115, USA. Phone: 617-919-2482; Fax: (617) 730 0528; raif.geha@ childrens.harvard.edu. 
TSLP protein consists of 121 amino acids with three potential $\mathrm{N}$-glycosylation sites and seven cysteine residues (2). The human TSLP gene is located on chromosome 5q22.1 next to the atopic cytokine cluster on $5 \mathrm{q} 31^{2}$. Both mouse and human TSLP exert their biological activities by binding to a heterodimeric receptor that consists of the IL-7 receptor $\alpha$-chain (IL-7R $\alpha$ ) and the TSLP receptor chain (TSLPR), which is closely related to the common receptor- $\gamma$ chain $(\gamma c)$. TSLPR has low affinity for TSLP, but in combination with IL-7R $\alpha$ generates a high affinity binding site for TSLP and triggers signaling (Figure 1). Despite the poor amino acid sequence identity between murine and human TSLP $(43 \%)$ and murine and human TSLPR (39\%), the fact that high affinity binding of TSLP to its receptor in both species requires IL-7R $\alpha$ suggest that human TSLP and TLPLR are orthologs to mouse TSLP and TSLPR ${ }^{3,4}$.

\section{Expression TSLP and TSLPR}

TSLP mRNA is expressed predominantly by epithelial cells in the thymus, lung, skin, intestine and tonsils as well as stromal cells and mast cells, but is not found in most hematopoietic cell types and endothelial cells ${ }^{2,5}$. TSLP is constitutively expressed in intestinal and thymic epithelial cells. Constitutive TSLP expression in intestinal epithelial cells (IEC) may be involved in tolerance to the commensal flora through the modulation of DC function ${ }^{5}$. TSLP that is constitutively expressed in the human thymus is responsible for the differentiation of $\mathrm{T}$ regulatory (Treg) cells by modulating the activity of thymic DCs ${ }^{6}$. The level of TSLP mRNA is dramatically up-regulated in mast cells after cross-linking high-affinity IgE receptors ${ }^{7}$.

TSLP protein is expressed in keratinocytes of acute and chronic skin lesions of atopic dermatitis (AD), but not in normal skin or non-lesional skin in AD (Figure 2) ${ }^{4}$. TSLP production in skin lesions is restricted to epidermal keratinocytes of the suprabasal layer and is absent from the undifferentiated basal layer. It also reported that human airway smooth muscle cells express constitutive level of TSLP in vitro and in vivo ${ }^{8}$. In contrast to the restricted expression of TSLP, TSLPR is more widely distributed. TSLPR mRNA has been detected on many immune cell types, including dendritic cells (DCs), T cells, B cells, mast cells, NKT cells and monocytes as well as in tissues from heart, skeletal muscle, kidney and liver. This suggests that TSLP can function on a broad range of cell types.

The regulation of TSLP expression in epithelial cells has not been fully elucidated. The fact that the presence and composition of the microflora affect the production of intestinal epithelial cell-derived TSLP suggests that exposure to microbial pathogen has a regulatory role in TSLP expression ${ }^{5}$. Recently, several studies have shown that TSLP can be induced by both exogenous stimuli including trauma, infection with microbes, Toll-like receptor (TLR) ligation and host-derived proinflammatory and Th2 cytokines. Allakhverdi et al reported that peptidoglycan, a component of gram-positive bacteria, induces the release of TSLP by human primary small airway epithelial cells, providing the first evidence of the role of pathogenic stimulation in the induction of TSLP ${ }^{9}$. The expression of TSLP was also found to be induced in both human and mouse airway epithelial cells exposed to pro-inflammatory mediators, including IL-1 $\beta$, TNF- $\alpha$, and selected TLR agonists (TLR2, TLR8, and TLR9 ligands). A nuclear factor $\mathrm{\kappa B}(\mathrm{NF \kappa B})$ site $3.7 \mathrm{~kb}$ upstream of the transcription start site of the TSLP gene promoter was shown to be critical for this inducible expression ${ }^{10}$. Both Th2 cytokines and TLR3 ligand (dsDNA) induce up-regulation of TSLP in primary human airway epithelial cells, which is dependent on NFKB and interferon regulatory factor 3 (IRF3) signaling through TLR3. Furthermore, infection of airway epithelial cells with rhinovirus, the most common respiratory virus that causes acute exacerbation of asthma, can lead to TSLP expression through stimulation with TLR3, which is enhanced synergistically by IL-4 and is inhibited by glucocorticoids ${ }^{11}$. Recent data showed that the long-form of two TSLP variants that arise by alternative splicing is highly induced by stimulation with TLR3 ligand, poly(I:C), on normal human bronchial epithelial cells (NHBE). Furthermore, a functional polymorphism 
(rs3806933) was identified in the promoter region of the long-form TSLP, which is associated with the allergic inflammation. This polymorphism creates a binding site for the transcription factor activating protein (AP)-1. The presence of this polymorphism was associated with poly (I:C) induced TSLP introduction by NHBE through enhancement of AP-1 binding to the promoter ${ }^{12}$. Given the fact that AP-1 expression is enhanced in the asthmatic airway, and reduced after glucocorticoid therapy ${ }^{13}$, the TSLP polymorphism that creates an AP-1 site may explain the inhibition of TSLP expression in response to glucocorticoid which blocks AP1 activity. Up-regulation of TSLP by proinflammatory and Th2 cytokines or TLR3 ligand also has been shown in cultured keratinocytes from healthy donors ${ }^{14}$. TLR3-mediated upregulation of TSLP expression on primary human airway epithelial cells and skin keratinocytes suggests a contribution of viral dsRNA to the local amplification of TSLP by Th2 cytokines in allergic inflammations. IL-25 and IL-33, two epithelial cell-derived cytokines can induce TSLP mRNA in epithelial cells from airway and gut, respectively ${ }^{15}$. In addition to acting on epithelial cells, the proinflammatory cytokines, IL-1 $\beta$, TNF- $\alpha$, but not Th2 cytokines, were found to induce TSLP mRNA and protein release from human airway smooth muscle cells ${ }^{8}$, TNF- $\alpha$ and ligands for TLR3 and TLR4 up-regulate TSLP production in synovial fibroblasts from patients with rheumatoid arthritis (RA) and osteoarthritis (OA) ${ }^{16,17}$. Damage or trauma to epithelial cells was reported to up-regulate TSLP expression (64). Recently, Demehri et al showed that general defects in differentiation/barrier formation of skin keratinocytes lead to up-regulation of TSLP expression both in vivo and in vitro ${ }^{18}$.

The mechanism by which TLSP expression is triggered upon allergen exposure remains to be investigated. A recent study showed that protease activity of papain, an occupational allergen homologous to the mite group 1 allergens, initiates $\mathrm{Th} 2$ sensitization in mice through activation of basophils to express Th2-inducing cytokines, including TSLP ${ }^{19}$. This reveals a new role of basophils, that migrate into draining lymph nodes in response to protease allergens, as a new source for TSLP, which provides the initial signal to promote Th2 differentiation in vivo. Mast cells up-regulate TSLP expression in nasal epithelium following the allergen challenge, as mast cell-deficient mice fail to up-regulate TSLP in a model of allergic rhinitis ${ }^{20}$.

The factors that negatively regulate TSLP expression are not known. IFN- $\gamma$, TGF- $\beta$, and IL-17 suppress the release of TSLP by human keratinocytes stimulated by poly I:C, a TLR3 ligand. However, factors regulating Th2 inflammation, such as IL-10, TGF- $\beta$, or IFN- $\gamma$, do not show an inhibitory effect on the release of TSLP from human skin explants stimulated by proinflammatory and Th2 cytokines ${ }^{14}$. The observation that defective intrinsic expression of TSLP in the IECs from mice with an IEC-specific deletion of IKK- $\beta$, the catalytic subunit of the IKK complex responsible for NF- $\kappa B$ activation, has indicated that TSLP might be regulated by IKK- $\beta$-dependent NF- $\kappa B$ activation within the intestinal microenvironment ${ }^{21}$. Nuclear receptors have been suggested to be involved in repressing TSLP expression in mouse keratinocytes, as ablation of retinoid $\times$ receptors (RXRs) in keratinocytes results in increased TSLP expression in these cells ${ }^{22}$. Furthermore, topical application of active vitamin D3 or the low-calcemic analogue MC903 leads to elevated TSLP production from normal keratinocytes 23. The RXR agonist 9-cis-retinoic acid (9-cis-RA) was shown to be a negative regulator of TSLP expression in airway epithelial cells through direct inhibition of NF- $\kappa B$ signaling, not through direct binding to the TSLP gene promoter ${ }^{24}$. However, whether inhibition of TSLP expression by RXR is NF- $\mathrm{KB}$-dependent is still controversial, because another group used more selective synthetic RXR or RAR agonists in mice for NF- $\mathrm{\kappa B}$ and TSLP gene expression analysis in the skin and obtained an opposite results ${ }^{25}$.

\section{TSLP signaling}

Both human and mouse TSLP-TSLPR interactions activate similar signaling pathways (Fig 1). Like IL-7, TSLP activates the transcription factor signal transducer and activator of 
transcription 3 (STAT3) in human and STAT5 in mouse and human, and induces the expression of common genes (such as Cish). However, the mechanisms that TLSP and IL-7 use to activate STAT5 are distinctly different. TSLP-mediated signal transduction does not require Jak1 and $\mathrm{Jak} 2$, as evidenced by the observation that dominant negative forms of Jak1 and Jak2 are unable to block TSLP-mediated STAT5 activation, in contrast, IL-7 signaling is inhibited by dominant negative Jak1 26 .

\section{Cellular targets of TSLP}

TSLP was originally discovered to be a potent factor for $\mathrm{B}$ and $\mathrm{T}$ cell growth and differentiation. The initial work on TSLP function mostly focused on its role on the maturation of immature B cells in mice. Subsequent several studies showed that human TSLP preferentially acts on myeloid cells such as monocytes and CD11 $\mathrm{c}^{+} \mathrm{DCs}$ in vitro. Detection of TSLPR expression on activated T cells and mast cells reveals a more complicated role of TSLP in immune system (Fig 1. Like IL-7, TSLP also exhibit species-specific functions and may function differently in humans and mice.

\section{TSLP and dendritic cells (DCs)}

DCs play a critical role in polarizing Th effector cells that play a role in the pathogenesis of various diseases depending on their specific expression of cytokines and co-stimulatory molecules and the influence of the individual tissue milieu from which they originate. Cytokines released at epithelial surface are likely to play an important role in influencing the ability of DCs to polarize T cells. Initially, human TSLP was found to potently enhance the maturation of the $\mathrm{CD} 11 \mathrm{c}^{+}$subset of DCs, as evidenced by the strong induction of the costimulatory molecules CD40 and CD80, as well as the release of the T cell-attracting chemokine, TARC and MDC, and of IL-8 and eotaxin-2, which attract neutrophils and eosinophils, respectively ${ }^{27}$. Further studies showed that DCs treated in vitro with TSLP fail to produce the Th1-polarizing cytokine IL-12 and up-regulate the expression of OX40 ligand (OX40L) (Figure 3). OX40L expression has been reported to be critical for the ability of TSLPtreated DCs to polarize naïve $\mathrm{T}$ cells to differentiate into inflammatory $\mathrm{Th} 2$ cells that produce Th2 cytokines, IL-4, IL-5, IL-13, and in addition TNF- $\alpha^{28}$. TSLP also has been shown to be a major regulatory cytokine for IL-12 production by DCs in the presence of other DCactivators. TSLP-treated DCs exhibit reduced production of IL-12/23p40 upon TLR ligation

${ }^{29}$, however, TSLP induces DCs to produce large amounts of IL-12 after CD40 ligand (CD40L) stimulation. More importantly, DCs activated with TSLP and CD40L induce naive CD4 ${ }^{+} \mathrm{T}$ cells to differentiate into effector T cells producing both Th1 and Th 2 cytokines ${ }^{30}$. Thus, TSLP could promote the persistence of Th2 inflammation even in the presence of IL-12-inducing signals via regulating the expression of IL-12 by DCs. Consistent with this notion, in vivo studies showed that the frequency of $\mathrm{CD}^{+} \mathrm{IL}^{-} 4^{+} \mathrm{T}$ cells in mice engineered to overexpress TSLP in keratinocytes under the control of the keratin 5 promotor (K5-TLSP transgenic mice) was dramatically increased in comparison to normal littermate controls ${ }^{31}$. There is also significantly less expression of IL- $4^{+}$cells and increased IFN- $\gamma^{+}$cells in the splenocytes of DO.11.10/TSLPR ${ }^{-1-}$ mice ${ }^{32}$. TSLP-mediated Th2 responses in the lung and skin of wild type (WT) mice are substantially inhibited by neutralization of OX40L in vivo ${ }^{33}$. TSLP expression by keratinocytes of patients with atopic dermatitis is associated with decreased Langerhans cells (LCs), a subset of DCs residing primarily in the epidermis, suggesting that TSLP causes LCs migration in situ ${ }^{4}$. Further study confirmed that TSLP acts on human LCs in the same way as on blood DCs, as migrating mature LCs induced by TSLP in a skin explant culture induce inflammatory Th2 cells and release TARC ${ }^{34}$.

In mice, the effect of TSLP on DCs remains unclear. It was initially reported that TSLP does not appear to have any biological effects on mouse DCs ${ }^{4}$, but it was later reported that it activates both bone marrow (BM)-derived DCs with a moderate increase in the expression 
level of MHCII and costimulatory molecules and the release of TARC. TSLP treatment also enhanced the expression of MHCII and costimulatory molecules on splenic DCs in response to antigen stimulation. Furthermore, TSLP-treated DCs support less IFN- $\gamma$ production by TCROVA transgenic DO11.10 CD4 ${ }^{+}$T cells in response to antigen ${ }^{32}$. Our studies demonstrated that TSLP enhances IL-4 and IL-13 secretion by the DO11.10 CD4 ${ }^{+} \mathrm{T}$ cells to a comparable extent in the presence of WT and TSLPR ${ }^{-/-}$DCs, but has no suppressive effect on IFN- $\gamma$ production ${ }^{35}$. Moreover, skin migrating $\mathrm{CD} 11 \mathrm{c}^{+} \mathrm{FITC}^{+}$DCs in draining lymph nodes of $\mathrm{TSLPR}^{-/-}$mice exhibit normal up-regulation of expression of MHC II and costimulatory molecules and support normally the production of Th2 cytokines by DO11.10 CD4 ${ }^{+}$cells in response to antigen. Furthermore, the systemic Th2 response to epicutaneous (EC) sensitization with ovalbumin (OVA) antigen was comparable in TSLPR ${ }^{-/}$and WT mice, as evidenced by comparable secretion of cytokines by splenocytes and comparable circulating levels of antiOVA antibodies ${ }^{35}$. This suggests that TSLP-TSLPR interactions may not be essential for the polarization of skin mouse DCs to induce a Th2 response in our model of allergic skin inflammation induced by EC sensitization. It is possible that cytokines and other molecules released in response to mechanical skin injury inflicted by tape stripping skew DCs to promote Th2 cell differentiation in the absence of a TSLP signal in this model ${ }^{35}$.

Compared with conventional DCs, intestinal DCs possess a more tolerogenic phenotype toward the microflora and food antigens. Although TSLP has been indicated to be a strong activator of human myeloid DCs that induces an inflammatory effector Th2 cells that express, in addition to classical Th 2 cytokines, the proinflammatory cytokine TNF- $\alpha$, IEC-conditioned resident mucosal DCs release IL-10 and IL-6, but not IL-12, and polarize T cells toward a protective Th2 response to defend against infection, but not towards an inflammatory Th1 response even after exposure to a microbial pathogen. Rimoldi et al found that TSLP is a critical component in conditioning DCs to induce "noninflammatory" effector Th2 cells producing the classical Th2 2 cytokines and the anti-inflammatory cytokine IL-10, suggesting that TSLP may contribute to local tolerance by induction of "noninflammatory" DCs in the intestine ${ }^{5}$. Furthermore, TSLP released by IECs was shown to enhance the production of a proliferation-inducing ligand (APRIL) via stimulating intestinal myeloid DC to induce IgA production by $\mathrm{B}$ cells ${ }^{36}$.

It has also been reported that TSLP activated human DCs contribute to homeostatic proliferation of naïve and memory $\mathrm{T}$ cells in the absence of foreign antigens. This could be mediated by the up-regulated expression of self peptide-MHC complexes and costimulatory molecules in DCs primed by TSLP ${ }^{37}$. The same group demonstrated that TSLP-conditioned DCs are critical in the maintenance of $\mathrm{Th} 2$ central memory $\left(\mathrm{T}_{\mathrm{CM}}\right) \mathrm{CD} 4^{+} \mathrm{T}$ cells homeostasis 38. These Th2 $\mathrm{T}_{\mathrm{CM}}$ cells could be further polarized to become Th2 effector memory cells by TSLP or other proallergic molecules, for example, IL-25, at the inflammatory sites ${ }^{39}$.

\section{TSLP and T cells}

It is widely accepted that IL-7 plays a critical nonredundant role in the development of T and $\mathrm{B}$ cells in mice and of $\mathrm{T}$ cells in humans ${ }^{40}$, as both $\mathrm{IL}-7^{-/-}$mice and IL-7 $\mathrm{R}^{-{ }^{-/}}$mice exhibit severely reduced thymic cellularity and defective T cell maturation. Since TSLP was originally identified as a growth factor in the supernatant of the Z210R.1 thymic stromal cell line and its heterodimeric receptor shares IL-7R $\alpha$ with IL-7, much initial attention was paid to the study of the possible role of TSLP in lymphocyte development. Early in vitro study showed mouse TSLP to be a potent cytokine for supporting T cell growth. The involvement of TSLP in T lymphopoiesis was further supported by the observation that more severe impairment of $\mathrm{T}$ cell development was seen in $\gamma_{\mathrm{c}}$ /TSLPR double-deficient mice over $\gamma_{\mathrm{c}}$ single-deficient mice, and by the finding that transgenically overexpressed TSLP was able to restore T cell development in IL- $7^{-1-}$ mice ${ }^{41,42}$. However, the TSLP-TSLPR pathway does not appear to be strictly required for $\mathrm{T}$ cell development under normal conditions, as $\mathrm{TSLPR}^{-1-}$ mice exhibit normal $\mathrm{T}$ 
cell development and normal distribution of $\mathrm{T}$ cell populations ${ }^{43}$. Mouse TSLP appears to have an effect on preferential expansion of $\mathrm{CD}^{+} \mathrm{T}$ cells, as addition of TSLP enhances the proliferative capacity of TCR-stimulated $\mathrm{CD}^{+} \mathrm{T}$ cells in vitro and $\mathrm{TSLPR}^{-/-} \mathrm{CD} 4^{+} \mathrm{T}$ cells expand less efficiently than WT CD4 ${ }^{+} \mathrm{T}$ cells in irradiated $\gamma_{\mathrm{c}} / \mathrm{Rag}^{-{ }^{-/}}$hosts ${ }^{41}$. Furthermore TSLP was found to act directly on naïve, but not memory $\mathrm{CD}^{+} \mathrm{T}$ cells, and to enhance the proliferation of naïve $\mathrm{CD} 4^{+} \mathrm{T}$ cells and their development into memory $\mathrm{T}$ cells in response to antigen (Figure 3$)^{32}$. Interestingly, human TSLP also can act on activated CD4 ${ }^{+} \mathrm{T}$ cells. In vitro studies demonstrated that TCR stimulation can induce the expression of TSLPR on $\mathrm{CD}^{+} \mathrm{T}$ cells, which leads to their responsiveness to TSLP treatment, as shown by enhanced proliferation of TCR-stimulated $\mathrm{CD} 4^{+} \mathrm{T}$ cells in the presence of TSLP ${ }^{44}$.

Although TSLP has been linked in many studies to the polarization of human Th2 cell differentiation through indirectly activating DCs, recent studies also demonstrated the ability of TSLP to drive Th2 differentiation through directly acting on $\mathrm{CD}^{+} \mathrm{T}$ cells in the mouse. Omori et al showed that in vitro TSLP treatment induces Th2 cell differentiation of naive $\mathrm{CD}^{+} \mathrm{T}$ cells in the absence of exogenous IL-4 and APCs through direct induction of endogenous IL-4 expression by $\mathrm{CD}^{+}{ }^{+} \mathrm{T}$ cell, as IL-4 blockade inhibits TSLP-mediated Th2 differentiation ${ }^{45}$. Our own data show that TSLP is able to enhance Th 2 cytokine secretion by antigen stimulated mouse $\mathrm{CD} 4^{+}$cells via a direct effect on $\mathrm{T}$ cells ${ }^{35}$. Thus, it is likely that TSLP plays multiple roles in promoting Th 2 cell differentiation and Th 2 cytokine production (Figure 3).

It reported that human TSLP promotes activation and differentiation of human CD8 ${ }^{+} \mathrm{T}$ cells into proallergic cytotoxic $\mathrm{T}$ cells that produce large amounts of IFN- $\gamma$ while retaining their capacity to produce IL-5 and IL- 13 by acting on DCs ${ }^{46}$. TSLP also can act directly on TCRactivated human and mouse $\mathrm{CD} 8^{+} \mathrm{T}$ cells that express TSLPR to augment their survival and promote up-regulation of the Bcl-2 antiapoptotic protein, but shows no effect on the homeostatic proliferation of $\mathrm{CD}^{+} \mathrm{T}$ cells ${ }^{47}$.

\section{TSLP and B cells}

The exact role of TSLP on B cell development remains unclear. In vitro studies initially showed that TSLP supports the differentiation of fetal liver and BM adult B cell progenitors into $\mathrm{IgM}^{+} \mathrm{B}$ cells and suggested that TSLP contributes to B lymphopoiesis possibly by mechanisms distinct from IL-7 ${ }^{1,48}$. Further studies found that TSLP is selectively active on pro-B cells derived from fetal liver, but not adult BM in vitro, despite the presence of fully functional TSLP receptors on both fetal and adult B cells ${ }^{49}$. In vivo studies have given conflicting results on the role of TSLP in B cell development. Vosshenrich et al. found 10-fold further reduction in B-cell progenitors and mature B cells in IL-7R $\alpha^{-/-}$mice compared with $\gamma_{\mathrm{c}}{ }^{-/-}$mice. This suggests that TSLP is the main factor driving IL-7-independent fetal and perinatal lymphopoiesis ${ }^{49}$. However, similar to T cell development, the examination of $\mathrm{TSLPR}^{-/}$mice revealed normal $\mathrm{B}$ cell development, including the $\operatorname{IgM}^{+}$compartment $^{43}$. Futhermore, using $\mathrm{TSLPR}^{-/-}$, IL- $7^{-/-}$and Flt3 $\mathrm{L}^{-/-}$mice, Jensen et al showed that Flt3L rather than TSLP is the key regulator of IL-7-independent B lymphopoiesis and that intact TSLP function is insufficient to restore detectable $\mathrm{B}$ lymphopoiesis in the absence of these two critical regulators of $\mathrm{B}$ cell progenitors 50 . Transgenic overexpression of TSLP in mice has resulted in opposite effects on B lymphopoiesis. Multilineage, ubiquitous overexpression of TSLP driven by the $\beta$-actin promoter results in suppressed B lymphopoiesis and enhanced myelopoiesis ${ }^{51}$, while a doxycycline-inducible, K5-TSLP transgene results in enhanced B cell lymphopoiesis, causing increases in pre-BCR ${ }^{+} \mathrm{B}$ cell subsets and expansion of all immature and mature $\mathrm{B}$ cell populations in the periphery ${ }^{52}$. Another group also found that epithelial specific overexpression of TSLP driven by the keratin 14 (K14) promoter restores all stages of B-cell differentiation in the BM of IL- $7^{-/-}$mice ${ }^{42}$. This discrepancy might be a result of different 
levels of systemic TSLP in transgenic mice and suggests that alterations in the circulating levels of TSLP can influence the frequency and composition of B cell populations in vivo. A recent study has shown that endogenously overexpressed or exogenous TSLP supplement during neonatal hematopoiesis results in drastic expansion of peripheral pre- and immature B cells, causing B cell lymphoproliferative disorder ${ }^{18}$.

\section{TSLP and Regulatory T (Treg) cells}

Treg cells were identified as a small population of $\mathrm{CD} 4^{+} \mathrm{CD} 25^{+} \mathrm{T}$ cells whose differentiation is under the control of the transcription factor forkhead box protein 3 (Foxp3). Treg cells have a critical suppressive function for maintenance of self-tolerance and prevention of immune responses. TSLP is found to be selectively expressed by epithelial cells of the Hassall's corpuscles (HCs), which is associated with an activated myeloid DC subpopulation in the human thymic medulla ${ }^{6}$. Watanabe et al showed that human TSLP promotes the differentiation of Foxp $3^{+}$regulatory $\mathrm{T}$ cells from $\mathrm{CD} 4^{+} \mathrm{CD} 8^{-} \mathrm{CD} 25^{-}$naïve thymocytes in a DC-dependent manner, as myeloid DCs isolated from human thymus stimulated with TSLP, but not with IL-7, $\mathrm{CD} 40 \mathrm{~L}$, or poly I:C, induce vigorous expansion of $\mathrm{CD} 4^{+} \mathrm{CD} 8^{-} \mathrm{CD} 25^{-}$thymocytes and Foxp3 by half of the expanded cells. They also showed that the ability of TSLP-DCs to induce the differentiation of $\mathrm{CD}^{+}{ }^{+} \mathrm{CD} 8^{-} \mathrm{CD} 25^{-}$thymocytes into Tregs depends on IL-2 and CD28 signaling ${ }^{6}$. Mice lack Hassal's corpuscles and TSLPR ${ }^{-/-}$mice have normal numbers of circulating $\mathrm{CD}^{+}{ }^{+} \mathrm{CD} 25^{+} \mathrm{Foxp} 3^{+}$Tregs. However, there are several studies to support a potential role for mouse TSLP in the direct promotion of Foxp $3^{+}$Tregs development in thymus and periphery. Culture of mouse fetal thymic organ or thymocytes with TSLP increased expression of Foxp $3^{+}$, suggesting that TSLP may act directly on thymocytes to induce Treg cell development in a DC-independent manner ${ }^{24,53}$. It also has been suggested that TSLP conditioned BM-derived DCs induce the conversion of naïve T-cells into functional $\mathrm{CD} 4^{+} \mathrm{CD} 25^{+} \mathrm{Foxp}^{+}$Treg cells, and that administration of exogenous TSLP or TSLP treated DCs protects the nonobese diabetic (NOD) mouse against diabetes, possibly by restoring $\mathrm{T}$ cell tolerance through induction of Treg cell differentiation and promotion of noninflammatory Th2 cells that produce IL-10 ${ }^{54}$. The precise role of TSLP in the development of natural Treg cells remains controversial, $\mathrm{TSLPR}^{-/-} \mathrm{DCs}$ from lamina propria have a similar ability to mediate extrathymic Treg cell development as WT DCs, suggesting that TSLP is not required for the development of inducible Treg cells ${ }^{55}$. The observation that combined deletion of receptors for IL-7 and TSLP greatly reduces thymic development, but not peripheral survival, of Treg cells, suggests that TSLP may not play a role in Treg cells homeostasis ${ }^{56}$.

\section{TSLP and innate immune cells}

TSLP can directly act on innate cells, including mast cells, basophils and NKT cells, to produce proinflamatory cytokines and Th2 cytokines, to initiate an innate phase of allergic inflammatory response or aggravate allergic inflammation in the absence of $\mathrm{T}$ lymphocytes and IgE antibodies. This is evidenced by the observations that K5-TSLP transgenic mice on TCR $\beta^{-1-}$ background develop skin inflammation characterized by epidermal thickening and dermal infiltrates that consist of mostly mast cells and eosinophils ${ }^{31}$. Furthermore, RAG1 ${ }^{-/-}$ mice treated with the vitamin D analog, MC903, exhibit dermal infiltration with eosinophils, mast cells, and DCs which is mediated by upregulated expressio of TSLP in the skin ${ }^{31}$. TSLP can directly activate human mast cells to produce proinflammatory Th2 cytokines and chemokines in the presence of IL- $1 \beta$ and TNF- $\alpha^{9}$. TSLP may potentially activate NKT cells to produce IL-13 and K5-TSLP transgenic mice that lack NKT cells fail to develop airway hyperreactivity in a mouse asthma model ${ }^{57}$. Thus, TSLP could contribute to Th2-mediated allergic responses through its ability to influence both innate and adaptive immune cells. 


\section{TSLP in disease}

TSLP has been suggested to be a master switch Th2 driven allergic inflammation in AD and asthma. Indeed, a number of studies have established a direct link between TLSP expression in skin keratinocytes and airway epithelial cells and the pathogenesis of atopic dermatitis and asthma, respectively. There is also evidence to suggest that TSLP may play a role in IBD and autoimmune disease.

\section{TSLP and allergic asthma}

TSLP has been implicated in the initiation of allergic airway inflammation. Elevated bronchial mucosal expression of TSLP has been associated with human asthma and correlates with disease severity ${ }^{58}$. TSLP is up-regulated in the lungs of mice in an OVA-driven model of airway inflammation ${ }^{59}$. TSLPR $^{-/-}$mice fail to develop airway inflammation in an OVAinduced model of allergic asthma ${ }^{32,59}$. Mice that express a lung-specific expression of a TSLP transgene driven by the surfactant protein C promoter (SPC-TSLP mice) develop a spontaneous, and progressive asthma-like disease characterized by leukocyte infiltration, goblet cell metaplasia, and airway hyperreactivity (AHR), as well as elevated serum IgE without overt sensitization and challenge with antigen. Elimination of Th2 cytokines, IL-4 and IL-13, by genetic targeting or inhibition by neutralizing antibody eliminated chronic lung inflammation spontaneously developed in SPC-TSLP mice, suggesting that TSLP-induced pathogenesis of asthma-like airway inflammation is mediated by intact Th2 responses ${ }^{60}$. Furthermore, using an acute model of TSLP-induced asthma, it has shown that TSLP alone was insufficient for the development of full airway inflammatory disease, although it induces innate responses including mucus overproduction and goblet cell metaplasia in the lung. Full developed airway inflammation requires the presence of foreign antigens and $\mathrm{CD} 4^{+} \mathrm{T}$ cells, Thus, TSLP may act as a susceptibility factor in the lung to generate aberrant Th2 allergic responses to antigen 61 .

\section{TSLP and Atopic dermatitis (AD)}

TSLP is expressed in keratinocytes of AD patients, but not healthy subjects or patients with nickel-induced contact dermatitis or cutaneous systemic lupus erythematosis ${ }^{4}$. This suggests a role of TSLP in Th2-mediated allergic skin inflammation. A link between TSLP expression and the pathogenesis of AD has been shown in several mouse models. K5-TLSP transgenic mice develop a AD-like dermatitis characterized by the development of eczematous lesions containing dermal inflammatory cell infiltrates, a dramatic increase in Th2 cells and elevated serum levels of IgE (Figure 4) ${ }^{22}$ Interestingly, selective ablation of RXRs in epidermal keratinocytes induces TSLP expression in epidermis and triggers an AD-like skin and system syndrome in adult mice. Induced expression of TSLP by keratinocytes is indespensible for the pathogenesis of an AD-like dermatitis triggered by topical application of MC903, a lowcalcemic analogue of vitamin D3 23,62 .

We showed that TSLPR ${ }^{-/-}$mice fail to develop allergic skin inflammation in a mouse model of AD elicited by repeated EC sensitization with OVA to tape-stripped skin. Local blockade of TSLP by a neutralizing antibody inhibits the development of allergic skin inflammation following skin challenge of immunized mice. This suggests that TSLP could amplify allergic skin inflammation in the effector phase by acting directly on skin infiltrating $\mathrm{T}$ cells to induce Th2 cytokine secretion (Fig 5).

$\mathrm{AD}$ is considered to be the initial step in the atopic march, since the majority of infants with AD develop asthma and allergic rhinitis in the later life. The induction of TSLP expression in mouse epidermal keratinocytes by topical application of MC903, accompanied by elevated circulating TSLP in the blood, was able to trigger an aggravation of OVA-induced allergic 
asthma phenotype ${ }^{9}$. TSLP overexpression by keratinocytes could be a risk factor for the development of allergic airway inflammation in AD patients.

\section{TSLP and Inflammatory bowel diseases (IBD)}

TSLP is constitutively expressed by primary IECs from healthy subjects but not from patients with Crohn disease ${ }^{5,21}$. In mouse, TSLP is constitutively expressed in IECs throughout the lower gastrointestinal (GI) tract, with the highest level of expression in the proximal large intestine ${ }^{5}$. Challenge with the intestinal nematode pathogen Trichuris leads to early upregulation of TSLP mRNA in the large intestine, indicating that TSLP mRNA expression may be affected by alterations in the abundance or composition of commensal bacterial populations in the GI tract and suggesting a possible regulatory function for IEC-derived TSLP in immune homeostasis within the large intestine ${ }^{5}$. Recent studies show that TSLP-TSLPR interactions are crucial for promoting protective Th2 immunity against Trichuris, which is mediated by Th2 cells ${ }^{29}$. After infection with Trichuris, mice with an IEC-specific deletion of IKK- $\beta$ exhibit a reduced expression of TSLP in the intestine and fail to develop a pathogen-specific CD4 ${ }^{+}$ Th2 response and to eradicate early infection ${ }^{21}$. Furthermore, disruption of TSLP-TSLPR interactions in vivo in TSLPR ${ }^{-/-}$mice or using neutralizing TSLP antibody leads to susceptibility to Trichuris infection, as evidenced that TSLPR ${ }^{-/-}$mice exhibit increased IFN$\gamma$ production and elevated worm burdens at day 21 after infection ${ }^{29}$. However, unlike mouse models of inflammation in the skin or the lung, TSLP is not required to initiate pathogenspecific Th2 cytokine responses in the intestine because neutralization of IFN- $\gamma$ antibodies restored pathogen-specific Th2 cytokine production and resulted in worm expulsion in Trichuris infected TSLPR ${ }^{-1-}$ mice $^{29}$. TSLPR $^{-/-}$mice also exhibited elevated expression of proinflammatory cytokines and early onset of intestinal inflammation in a mouse model of colitis induced by dextran sodium sulfate (DSS), suggesting a general anti-inflammatory role for TSLP in the GI tract ${ }^{29}$.

\section{TSLP and antoimmune disease}

There is evidence to suggest that TSLP is involved in autoimmune diseases, The exact role in the pathophysiology these diseasess remains unclear. Overexpression of TSLP driven by $L c k$ gene that encodes a lymphocyte-specific protein-tyrosine kinase, $\mathrm{p} 56^{l c k}$, in mice results in develop cryoglobulinemia with high systemic levels of IgM and IgG and a systemic inflammatory disease, including a kidney disease closely resembling human cryoglobulinassociated membranoproliferative glomerulonephritis ${ }^{63}$. K5-TSLP transgenic mice develop cryoglobulins that are composed of both polyclonal $\operatorname{IgM}$ and $\operatorname{IgG}$ and their deposition in the kidney ${ }^{52}$. These observations indicate that TSLP may promote humoral autoimmunity by enhancing B cell population expansion and/or higher antibody production. Increased levels of TSLP as well as TNF- $\alpha$ were found in synovial fluid specimens derived from patients with RA. TNF- $\alpha$ induces TSLP production by synovial fibroblasts from patients with RA ${ }^{17}$. Inhibition of TSLP by neutralizing antibody ameliorates joint inflammation in a TNF- $\alpha$ dependent mouse model of arthritis induced by anti-type II collagen suggesting that TSLP may be involved in the pathogenesis of inflammatory arthritis ${ }^{17}$.

\section{Conclusion}

The evidence available to date indicates that TSLP plays a critical role in Th2 cell differentiation. This role is exerted both at the induction phase of the Th2 response via polarization of DCs to drive Th2 cell differentiation and at the effector phase of the response by promoting the expansion of activated T cells and their secretion of Th2 cyokines. More importantly, there is strong evidence that the Th2 skewing properties of TSLP play an important role in the pathogenesis of $\mathrm{AD}$ and asthma, two diseases that affect a large segment of the population, and which are on the rise, Conversely, TSLP appears to protect against IBD, a 
disease dominated by Th17 and Th1 driven inflammation. TSLP is a good therapeutic target in the treatment of allergic diseases, but its protective role in IBD sounds a note of caution because neutralization of TSLP could potentially unmask or aggravate Th17 and or Th2 dominated inflammatory disease.

\section{Acknowledgments}

This work was supported by USPHS grant AR-047417 and Atopic Dermatitis Vaccinia Network contract N01 AI40030.

\section{References}

1. Friend SL, Hosier S, Nelson A, et al. Exp Hematol 1994;22(3):321. [PubMed: 8112430]

2. Quentmeier H, Drexler HG, Fleckenstein D, et al. Leukemia 2001;15(8):1286. [PubMed: 11480573]

3. Pandey A, Ozaki K, Baumann H, et al. Nature immunology 2000;1(1):59. [PubMed: 10881176] Park LS, Martin U, Garka K, et al. The Journal of experimental medicine 2000;192(5):659. [PubMed: 10974032]

4. Soumelis V, Reche PA, Kanzler H, et al. Nature immunology 2002;3(7):673. [PubMed: 12055625]

5. Rimoldi M, Chieppa M, Salucci V, et al. Nature immunology 2005;6(5):507. [PubMed: 15821737]

6. Watanabe N, Wang YH, Lee HK, et al. Nature 2005;436(7054):1181. [PubMed: 16121185]

7. Okayama Y, Okumura S, Sagara H, et al. Eur Respir J. 2009

8. Zhang K, Shan L, Rahman MS, et al. Am J Physiol Lung Cell Mol Physiol 2007;293(2):L375. [PubMed: 17513456]

9. Allakhverdi Z, Comeau MR, Jessup HK, et al. The Journal of experimental medicine 2007;204(2): 253. [PubMed: 17242164]

10. Lee HC, Ziegler SF. Proc Natl Acad Sci U S A 2007;104(3):914. [PubMed: 17213320]

11. Kato A, Favoreto S Jr, Avila PC, et al. J Immunol 2007;179(2):1080. [PubMed: 17617600]

12. Harada M, Hirota T, Jodo AI, et al. Am J Respir Cell Mol Biol 2009;40(3):368. [PubMed: 18787178]

13. Demoly P, Basset-Seguin N, Chanez P, et al. Am J Respir Cell Mol Biol 1992;7(2):128. [PubMed: 1353973] Demoly P, Chanez P, Pujol JL, et al. Respir Med 1995;89(5):329. [PubMed: 7638366]

14. Bogiatzi SI, Fernandez I, Bichet JC, et al. J Immunol 2007;178(6):3373. [PubMed: 17339431] Kinoshita H, Takai T, Le TA, et al. J Allergy Clin Immunol 2009;123(1):179. [PubMed: 19056108]

15. Angkasekwinai P, Park H, Wang YH, et al. The Journal of experimental medicine 2007;204(7):1509. [PubMed: 17562814] Humphreys NE, Xu D, Hepworth MR, et al. J Immunol 2008;180(4):2443. [PubMed: 18250453]

16. Ozawa T, Koyama K, Ando T, et al. Modern rheumatology / the Japan Rheumatism Association 2007;17(6):459. [PubMed: 18084696]

17. Koyama K, Ozawa T, Hatsushika K, et al. Biochemical and biophysical research communications 2007;357(1):99. [PubMed: 17416344]

18. Demehri S, Liu Z, Lee J, et al. PLoS Biol 2008;6(5):e123. [PubMed: 18507503]

19. Sokol CL, Barton GM, Farr AG, et al. Nature immunology 2008;9(3):310. [PubMed: 18300366]

20. Miyata M, Hatsushika K, Ando T, et al. Eur J Immunol 2008;38(6):1487. [PubMed: 18461563]

21. Zaph C, Troy AE, Taylor BC, et al. Nature 2007;446(7135):552. [PubMed: 17322906]

22. Li M, Messaddeq N, Teletin M, et al. Proc Natl Acad Sci U S A 2005;102(41):14795. [PubMed: 16199515]

23. Li M, Hener P, Zhang Z, et al. J Invest Dermatol 2009;129(2):498. [PubMed: 18650845]

24. Lee HC, Headley MB, Iseki M, et al. J Immunol 2008;181(8):5189. [PubMed: 18832669]

25. Gericke J, Gamlieli A, Weiss K, et al. J Immunol 2009;182(1):3. author reply 3. [PubMed: 19109125]

26. Isaksen DE, Baumann H, Trobridge PA, et al. J Immunol 1999;163(11):5971. [PubMed: 10570284]

27. Reche PA, Soumelis V, Gorman DM, et al. J Immunol 2001;167(1):336. [PubMed: 11418668]

28. Ito T, Wang YH, Duramad O, et al. The Journal of experimental medicine 2005;202(9):1213. [PubMed: 16275760] 
29. Taylor BC, Zaph C, Troy AE, et al. The Journal of experimental medicine 2009;206(3):655. [PubMed: 19273626]

30. Watanabe N, Hanabuchi S, Marloie-Provost MA, et al. Blood 2005;105(12):4749. [PubMed: 15741223]

31. Yoo J, Omori M, Gyarmati D, et al. The Journal of experimental medicine 2005;202(4):541. [PubMed: 16103410]

32. Al-Shami A, Spolski R, Kelly J, et al. The Journal of experimental medicine 2005;202(6):829. [PubMed: 16172260]

33. Seshasayee D, Lee WP, Zhou M, et al. J Clin Invest 2007;117(12):3868. [PubMed: 18060034]

34. Ebner S, Nguyen VA, Forstner M, et al. J Allergy Clin Immunol 2007;119(4):982. [PubMed: 17320941]

35. He R, Oyoshi MK, Garibyan L, et al. Proc Natl Acad Sci U S A 2008;105(33):11875. [PubMed: 18711124]

36. He B, Xu W, Santini PA, et al. Immunity 2007;26(6):812. [PubMed: 17570691]

37. Watanabe N, Hanabuchi S, Soumelis V, et al. Nature immunology 2004;5(4):426. [PubMed: 14991051]

38. Wang YH, Ito T, Wang YH, et al. Immunity 2006;24(6):827. [PubMed: 16782037]

39. Wang YH, Angkasekwinai P, Lu N, et al. The Journal of experimental medicine 2007;204(8):1837. [PubMed: 17635955]

40. von Freeden-Jeffry U, Vieira P, Lucian LA, et al. The Journal of experimental medicine 1995;181 (4):1519. [PubMed: 7699333] Puel A, Ziegler SF, Buckley RH, et al. Nat Genet 1998;20(4):394. [PubMed: 9843216]

41. Al-Shami A, Spolski R, Kelly J, et al. The Journal of experimental medicine 2004;200(2):159. [PubMed: 15263024]

42. Chappaz S, Flueck L, Farr AG, et al. Blood 2007;110(12):3862. [PubMed: 17702899]

43. Carpino N, Thierfelder WE, Chang MS, et al. Mol Cell Biol 2004;24(6):2584. [PubMed: 14993294]

44. Rochman I, Watanabe N, Arima K, et al. J Immunol 2007;178(11):6720. [PubMed: 17513717]

45. Omori M, Ziegler S. J Immunol 2007;178(3):1396. [PubMed: 17237387]

46. Gilliet M, Soumelis V, Watanabe N, et al. The Journal of experimental medicine 2003;197(8):1059. [PubMed: 12707303]

47. Rochman Y, Leonard WJ. J Immunol 2008;181(11):7699. [PubMed: 19017958]

48. Ray RJ, Furlonger C, Williams DE, et al. Eur J Immunol 1996;26(1):10. [PubMed: 8566050] Levin SD, Koelling RM, Friend SL, et al. J Immunol 1999;162(2):677. [PubMed: 9916685]

49. Vosshenrich CA, Cumano A, Muller W, et al. Nature immunology 2003;4(8):773. [PubMed: 12872121]

50. Jensen CT, Kharazi S, Boiers C, et al. Nature immunology 2007;8(9):897. author reply 898. [PubMed: 17712336]

51. Osborn MJ, Ryan PL, Kirchhof N, et al. Blood 2004;103(3):843. [PubMed: 14512322]

52. Astrakhan A, Omori M, Nguyen T, et al. Nature immunology 2007;8(5):522. [PubMed: 17401368]

53. Jiang Q, Su H, Knudsen G, et al. BMC immunology 2006;7:6. [PubMed: 16579866]

54. Besin G, Gaudreau S, Menard M, et al. Diabetes 2008;57(8):2107. [PubMed: 18477807]

55. Sun CM, Hall JA, Blank RB, et al. The Journal of experimental medicine 2007;204(8):1775. [PubMed: 17620362]

56. Mazzucchelli R, Hixon JA, Spolski R, et al. Blood 2008;112(8):3283. [PubMed: 18664628]

57. Nagata Y, Kamijuku H, Taniguchi M, et al. International archives of allergy and immunology 2007;144(4):305. [PubMed: 17652941]

58. Ying S, O'Connor B, Ratoff J, et al. J Immunol 2008;181(4):2790. [PubMed: 18684970]

59. Zhou B, Comeau MR, De Smedt T, et al. Nature immunology 2005;6(10):1047. [PubMed: 16142237]

60. Zhou B, Headley MB, Aye T, et al. J Immunol 2008;181(9):6557. [PubMed: 18941246]

61. Headley MB, Zhou B, Shih WX, et al. J Immunol 2009;182(3):1641. [PubMed: 19155513]

62. Li M, Hener P, Zhang Z, et al. Proc Natl Acad Sci U S A 2006;103(31):11736. [PubMed: 16880407] 
63. Taneda S, Segerer S, Hudkins KL, et al. Am J Pathol 2001;159(6):2355. [PubMed: 11733384] 


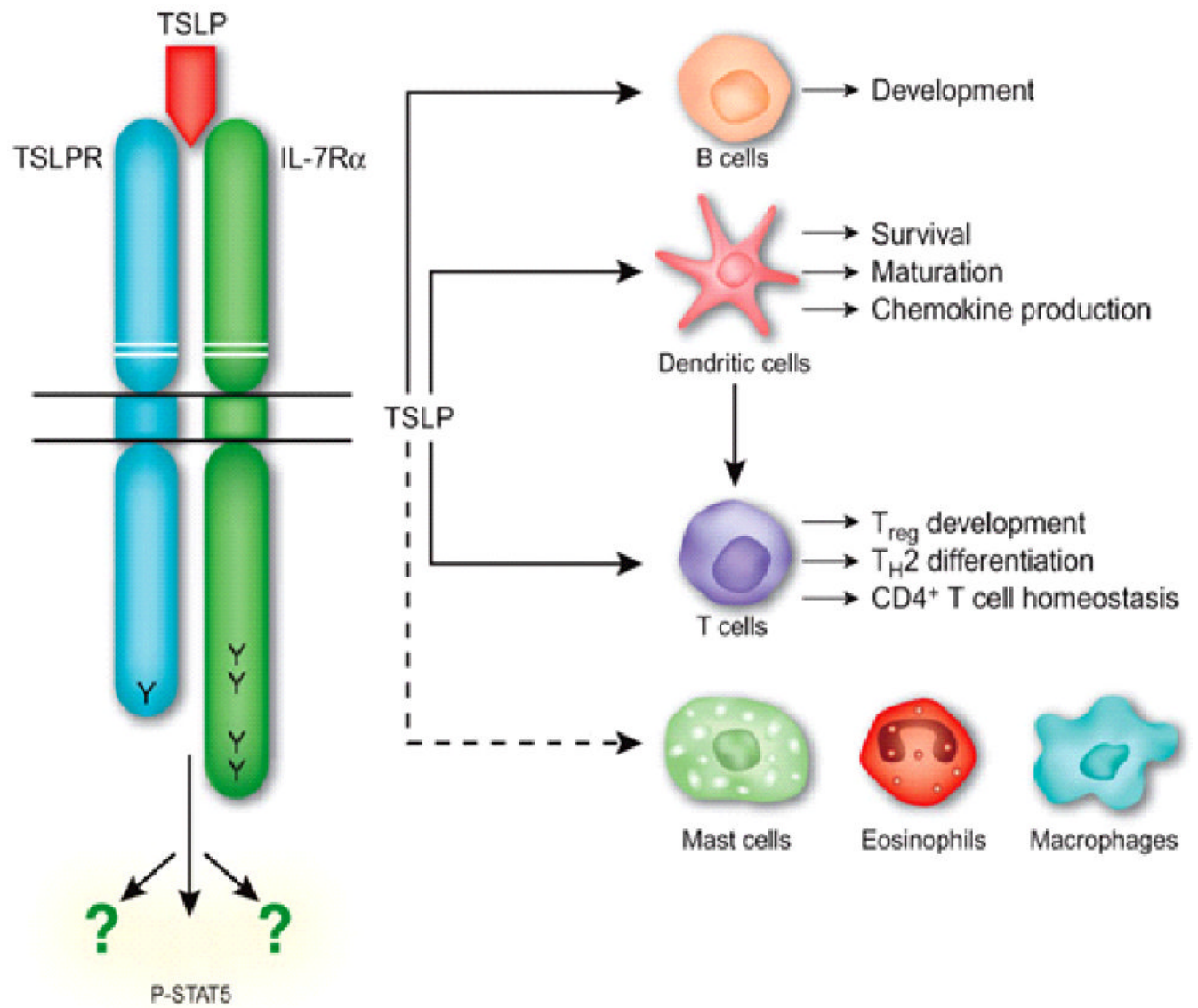

Figure 1.

Structure of the heterodimeric TSLP receptor and the its cellular targets immune cells. TSLPR signals via activation and phosphorylation of STAT5 (P-STAT5) and other unidentified pathways. Adapted from Nature Immunol. 2009; 7: 709-714 

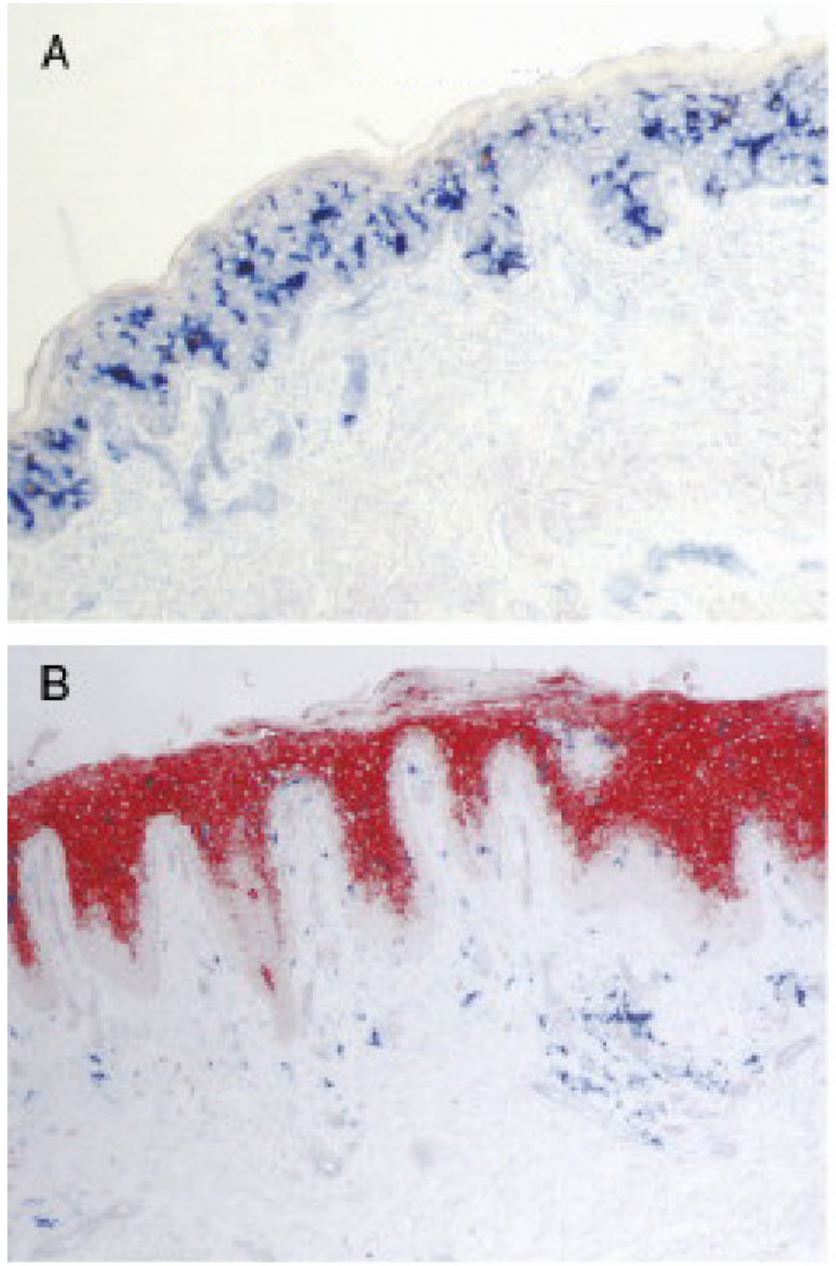

Figure 2.

TSLP expression in epidermis of skin from patients with atopic dermatitis but not from healthy control. (A) No detectable TSLP expression (no red staining). in epidermis from skin of health control. (B) High expression of TSLP (red staining) in epidermis from skin lesion of atopic dermatitis. Adapted from Adv Immunol. 2009; 101:1-25 


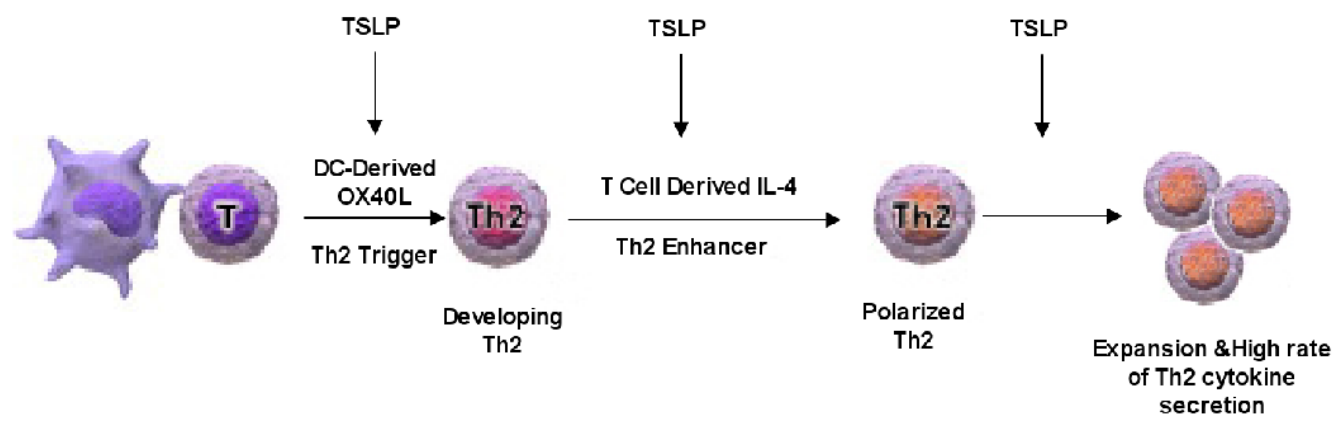

Figure 3.

TSLP acts through multiple pathways in Th2 cell differentiation and Th2 cytokine production. Adapted from Journal of Allergy and Clinical Immunology, 2007;120 (2): 238-244. 


\section{NLC}
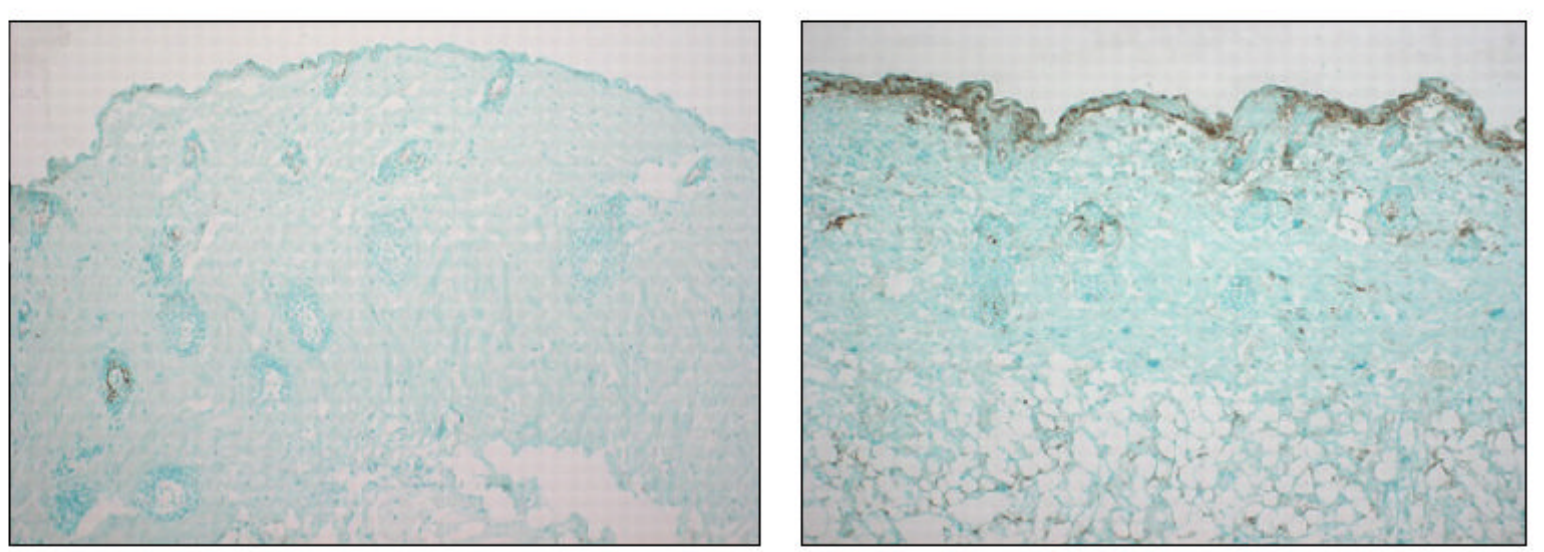
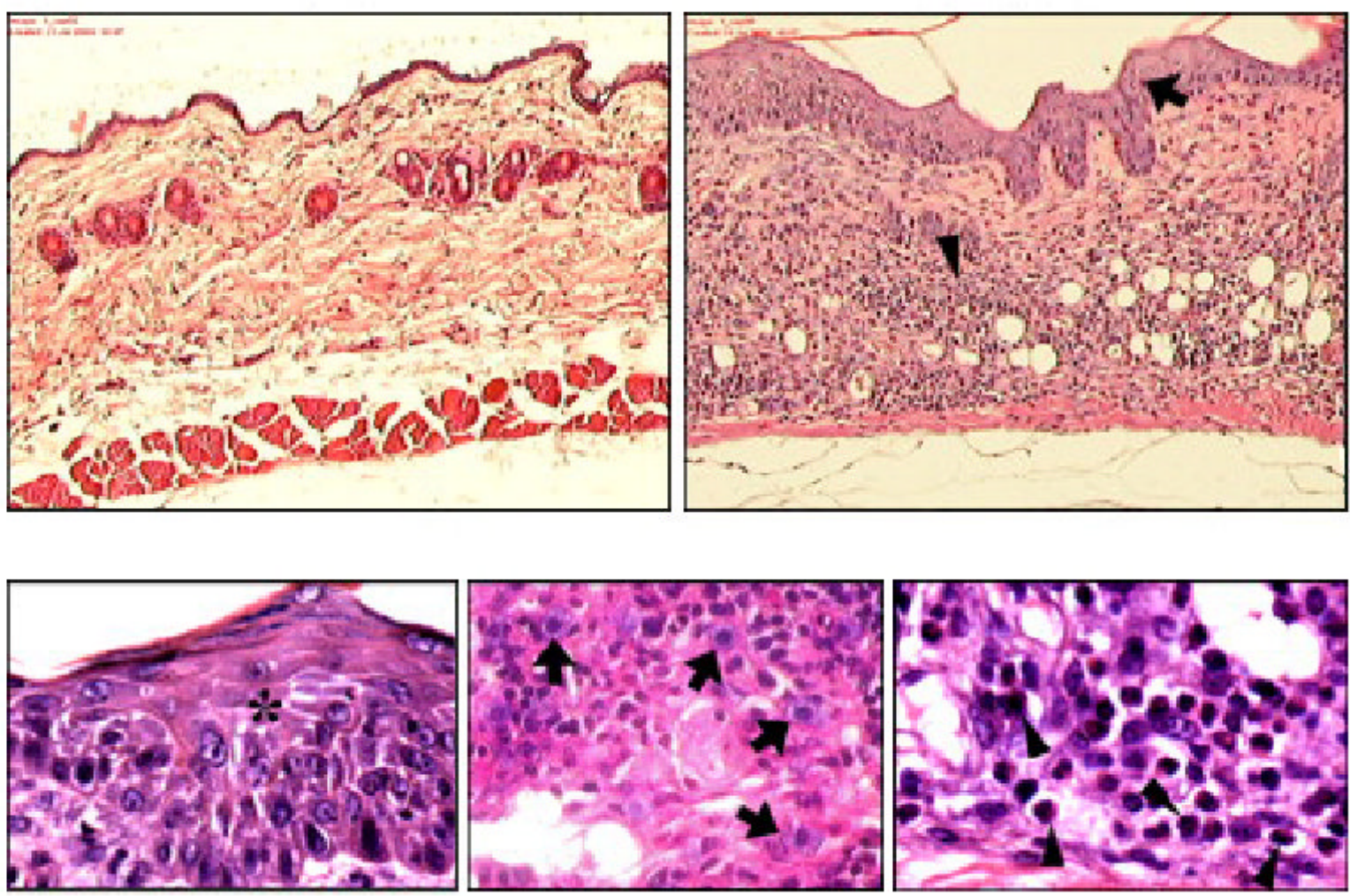

Figure 4.

K5-TLSP transgenic mice develop a AD-like dermatitis. (A). Immunohistochemical staining showing TSLP expression (brown) in the epidermis and its basement membrane from skin of a K5-TSLP mouse as compared with normal littermate control (NLC). (B) Skin sections stained with H\&E showing pronounced acanthosis (arrow) and dermal infiltration by mast cells (arrows) and eosinophils (arrowheads) at 100x. 

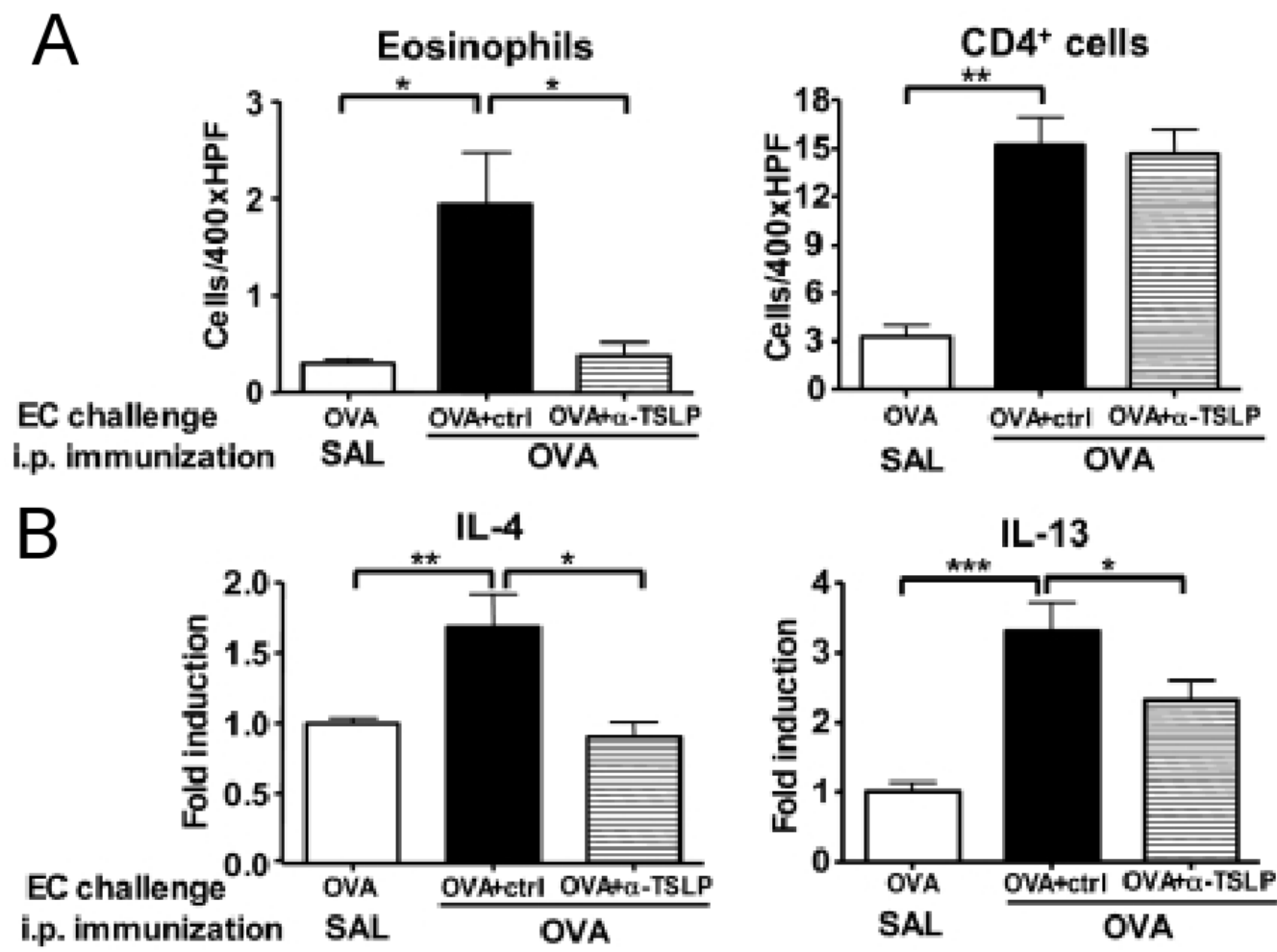

Figure 5.

Attenuated skin inflammation in OVA-challenged skin of i.p. immunized mice treated with neutralizing antibodies to TSLP compared with those treated with isotype control. (A) Number of infiltrating eosinophils and CD4+ cells. (B) expression of mRNA for IL-4 and IL-13. Proc Natl Acad Sci U S A. 2008; 105(33):11875-80 